\title{
What a nostril knows: Olfactory nerve-evoked AMPA responses increase while NMDA responses decrease at 24-h post-training for lateralized odor preference memory in neonate rat
}

\author{
Qi Yuan ${ }^{1}$ and Carolyn W. Harley ${ }^{2,3}$ \\ ${ }^{1}$ Biomedical Sciences, Faculty of Medicine, and ${ }^{2}$ Department of Psychology, Faculty of Science, Memorial University of Newfoundland, \\ St. John's, NL, Canada A1B $3 \times 9$
}

\begin{abstract}
Increased AMPA signaling is proposed to mediate long-term memory. Rat neonates acquire odor preferences in a single olfactory bulb if one nostril is occluded at training. Memory testing here confirmed that only trained bulbs support increased odor preference at $24 \mathrm{~h}$. Olfactory nerve field potentials were tested at $24 \mathrm{~h}$ in slices from trained and untrained bulbs. A larger AMPA component and a smaller NMDA component characterized responses in the bulb receiving odor preference training. Field potential changes were not seen in a bulbar region separate from the lateral odor-encoding area. These results support models in which memory is mediated by increased olfactory nerve-mitral cell AMPA signaling, and memory stability is promoted by decreased NMDA-mediated signaling.
\end{abstract}

Increases in fast excitatory transmission in informational pathways through increases in glutamatergic AMPA receptor connectivity have been proposed to underlie the network changes that support learning and memory (Malinow and Malenka 2002). Such changes can be recruited by the activity of glutamatergic NMDA receptors, which act as coincidence detectors when a "to be conditioned" glutamatergic sensory input is related to strong depolarizing post-synaptic events, or intracellular signaling events that promote the opening of NMDA receptor channels (Debanne 1996; Cain 1997). NMDA receptors are hypothesized to initiate plasticity in learning and memory networks, while AMPA receptors underlie the informational or connectional changes. NMDAinitiated plasticity mechanisms appear to down-regulate with age and may account for developmental and activity-dependent changes in plasticity (Isaac et al. 1997; Rumpel et al. 1998; Franks and Isaacson 2005). A reduction in NMDA-mediated plasticity mechanisms could, theoretically, assist the stability of long-term memories (Philpot 2005).

In the rat pup odor-preference conditioning model we have suggested that, as for other learning and plasticity models, AMPA receptor increases mediate the 24 -h memory produced in a single 10-min session of odor paired with an effective unconditioned stimulus, such as stroking or i.p. isoproterenol on postnatal day 6 (PD 6). This hypothesis has been supported by an increase in membrane AMPA receptors $24 \mathrm{~h}$ after training, as revealed in synaptoneurosome preparations from trained pups compared with controls and in increases in AMPA immunohistochemical staining densities at the same time point in the glomerular layer, where the olfactory nerve contacts mitral cell dendrites, in trained versus untrained olfactory bulbs (Cui et al. 2011). AMPA receptor insertion in the membrane appears causally linked to odor-preference memory, since infusion of an interference peptide that prevents AMPA insertion also prevents memory expression at $24 \mathrm{~h}$ (Cui et al. 2011). Others have reported that NMDA

\footnotetext{
${ }^{3}$ Corresponding author.

E-mail charley@play.psych.mun.ca.

Article is online at http://www.learnmem.org/cgi/doi/10.1101/lm.024844.111.
}

receptor contributions decrease developmentally in sensory systems, including the olfactory system, as measured at lateral olfactory tract synapses in the piriform cortex (Franks and Isaacson 2005). These changes are proposed to be related to reductions in plasticity as a function of experience and development. Here, we unilaterally condition a single olfactory bulb on PD 6 by using nostril occlusion during the training paradigm. Subsequent testing of pups with either the trained or untrained nostril verifies previous reports of lateralized odor-preference memories with single nostril training (Kucharski et al. 1986; Kucharski and Hall 1987, 1988). We then directly measure the field responses to olfactory nerve stimuli in vitro of slices from pairs of trained and untrained olfactory bulbs $24 \mathrm{~h}$ after training.

Sprague Dawley rat pups (Charles River) of both sexes were used in this study. Day of birth was considered to be PD 0 and litters were culled to 12 pups on PD 1. Dams were maintained under a 12-h reverse light/dark cycle at $22^{\circ} \mathrm{C}$ in polycarbonate cages with ad libitum access to food and water. All experimental procedures were approved by the Institutional Animal Care Committee at Memorial University of Newfoundland and follow the guidelines set by the Canadian Council on Animal Care.

All pups were trained with left nostril occluded on PD 6. Twenty four hours later, pups were tested for odor preference with either the left or right nostril occluded. Three groups were included in the study (Fig. 1A). (1) O/S LL: pups trained with Odor + Stroking, and their left nostrils occluded during both training and testing; (2) O/S LR: pups trained with Odor + Stroking, and their left nostrils occluded during training, but with right nostrils occluded during testing; (3) O/O LL: pups exposed to odor only, and their left nostrils occluded during both training and testing.

On PD6, rat pups were removed from the dam and put on fresh bedding 10 min before odor exposure. Right before training, the left nostril of the pup was blocked with odorless silicone grease. Pups were assigned to one of two conditions: (1) Odor + Stroking $(\mathrm{O} / \mathrm{S})$ or $(2)$ Odor Only $(\mathrm{O} / \mathrm{O})$. In the $\mathrm{O} / \mathrm{S}$ group, pups were placed on peppermint-scented bedding $(0.3 \mathrm{~mL}$ of peppermint extract per $500 \mathrm{~mL}$ of clean bedding) in an acrylic training 
A

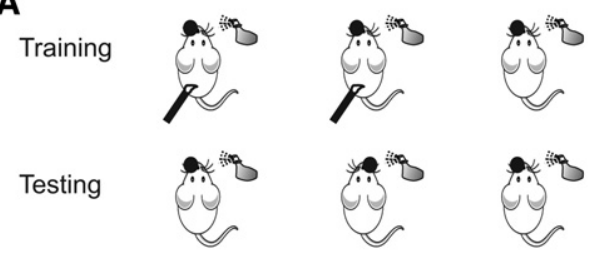

B

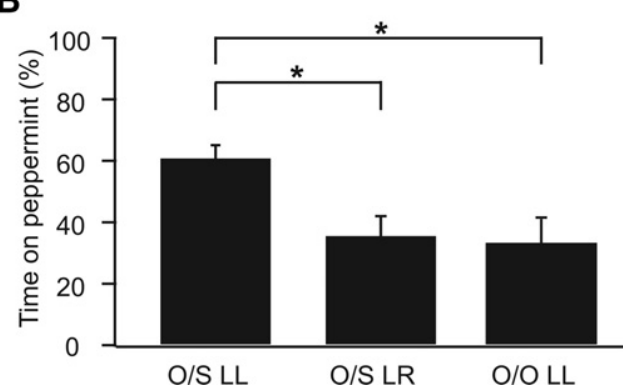

Figure 1. Early odor-preference learning can be acquired through one nostril. (A) Schematic of the odor training and testing in rat pups. Three groups were included in the study: an "O/S LL" group that underwent Odor + Stroking training and odor-preference testing with the same nostril (left) occluded; an "O/S LR" group in which the pups' left nostrils were occluded during Odor + Stroking training, while testing was conducted with the opposite (right) nostril occluded; an "O/O LL" control group in which pups were exposed to odor only during training and the left nostrils were occluded during both training and testing. Dots indicate sides of occlusion. Perfume bottles represent the Odor. Brushes represent Stroking. (B) Percentage time of pups in different groups spent on peppermint-scented bedding. $\left(^{*}\right) P<0.05$.

box $\left(20 \times 20 \times 5 \mathrm{~cm}^{3}\right)$ and stroked vigorously on the hind region using a sable brush every other $30 \mathrm{sec}$ for $30 \mathrm{sec}$ over a 10 -min period. In the $\mathrm{O} / \mathrm{O}$ group, the pups were exposed to the peppermint-scented bedding without being stroked. After training, the silicone grease was removed and the pups were returned to the dams.

On PD 7, each pup was tested for odor-preference memory. A stainless steel box $(30 \times 20 \times 18 \mathrm{~cm})$ was placed on top of two training boxes separated by a $2-\mathrm{cm}$ neutral zone. One box contained peppermint-scented bedding, while the other box contained clean, unscented bedding. Each pup was removed from the nest and either their left or right nostril was occluded with super gel glue according to the assignment described above. Super gel glue required less monitoring than the silicone grease used during training. For testing, pups underwent five separate 1-min trials, during which they were placed in the neutral zone of the test box and allowed to move freely. After each trial the pup was removed from the test box for a 1-min intertrial interval. During each test trial, when the pup's nose moved from the neutral zone to either the peppermint side or the unscented side, the experimenter began recording time. The total amount of time spent over peppermint-scented bedding and unscented bedding over all five trials was calculated separately. Values reported are the percentages of time animals spent over the peppermintscented bedding divided by the total time spent over peppermint + unscented bedding combined. Data are mean \pm SEM. A one-way ANOVA was used to determine statistical significance.

As shown in Figure 1, $24 \mathrm{~h}$ later, pups that underwent Odor + Stroking training demonstrated learning when the trained nostrils were open during testing $(\mathrm{O} / \mathrm{S} \mathrm{LL}, 61.06 \pm 4.12 \%$ on the peppermint side, $n=8$ ) (Fig. 1B), compared with pups with the untrained nostril open during testing $(\mathrm{O} / \mathrm{S}$ LR, $35.75 \pm 6.38 \%$, $n=8)$ or pups that were previously exposed to odor only $(\mathrm{O} / \mathrm{O}$
LL, $33.35 \pm 8.16 \%, n=8$ ) and then tested with the nostril of exposure. The one-way ANOVA demonstrated a significant difference among the groups $\left(F_{(2,23)}=5.69, P=0.01\right)$; while post hoc Tukey tests showed a significant difference between the O/S LL and $\mathrm{O} / \mathrm{S}$ LR groups $(q=3.93, P=0.03)$, as well as between $\mathrm{O} / \mathrm{S}$ LL and O/O LL groups $(q=4.31, P=0.02)$.

For the ex vivo testing of the physiological changes induced by training $24 \mathrm{~h}$ later, PD 5-8 rats were subject to either the Odor + Stroke or Odor Only training, with the left nostril again occluded with silicone grease right before training and removed right after. Twenty-four hours later, pups were anesthetized with halothane inhalation and then decapitated. Horizontal olfactory bulb slices were cut at $400 \mu \mathrm{m}$ with a vibrotome (Leica VT 1000P) in ice-cold high-sucrose aCSF containing the following (in millimolars): $83 \mathrm{NaCl}, 2.5 \mathrm{KCl}, 0.5 \mathrm{CaCl}_{2}, 3.3 \mathrm{MgSO}_{4}, 1 \mathrm{NaH}_{2} \mathrm{PO}_{4}$, $26.2 \mathrm{NaHCO}_{3}, 22$ glucose, and 72 sucrose equilibrated with $95 \%$ $\mathrm{O}_{2}$ and $5 \% \mathrm{CO}_{2}$. Slices were hemisected and those from occluded and nonoccluded trained olfactory bulbs were separated before incubation at $34^{\circ} \mathrm{C}$ for $30 \mathrm{~min}$ in the same high-sucrose aCSF. Slices were then left at room temperature until use. During recording, slices were superfused with aCSF containing the following (in millimolars): $119 \mathrm{NaCl}, 2.5 \mathrm{KCl}, 2.5 \mathrm{CaCl}_{2}, 1.3 \mathrm{MgSO}_{4}, 1$ $\mathrm{NaH}_{2} \mathrm{PO}_{4}, 26.2 \mathrm{NaHCO}_{3}, 22$ glucose equilibrated with $95 \% \mathrm{O}_{2}$ and $5 \% \mathrm{CO}_{2}$, and viewed with an upright microscope (Olympus BX51) using differential interference contrast (DIC) optics.

Extracellular field potential recordings were obtained with glass pipettes filled with aCSF $(1-2 \mathrm{M} \Omega)$ and positioned at the glomerular layer in the mid-lateral region of the olfactory bulb slice (Fig. 2C,E). It has been shown in previous reports that this midlateral glomerular region represents the "hot spot" of peppermint enhanced by odor learning (Johnson and Leon 1996). Additional control experiments were performed with recording in the medial region of the olfactory bulb slices from animals that underwent the same training (Fig. 2D). The stimulation electrode was placed at the ON layer adjacent to the glomeruli that were recorded. The ON was stimulated by a single test stimulus at incrementing stimulation intensities $(20-100 \mu \mathrm{A})$ using a concentric bipolar stimulating electrode (FHC). After recording in aCSF, slices were perfused with low $\mathrm{Mg}^{2+}(100 \mu \mathrm{M})$ aCSF and NBQX $(20 \mu \mathrm{M})$ to block the AMPA component of the fEPSP and to isolate the NMDA component. We have found that lowering $\mathrm{Mg}^{2+}$ to $100 \mu \mathrm{M}$ enhances the NMDA component of the fEPSP in most cases, but avoids the epileptic effect sometimes caused by $\mathrm{Mg}^{2+}$ free solutions. The recording was repeated with the same series of ON stimulations. All experiments were conducted at 30$32^{\circ} \mathrm{C}$. Electrophysiological data were recorded with a Multiclamp $700 \mathrm{~B}$ (Molecular Devices), filtered at $2 \mathrm{kHz}$ and digitized at $10 \mathrm{kHz}$. Data acquisition and analyses were performed with pClamp10 (Molecular Devices) and Igor Pro 6.10A (WaveMetrics).

As shown in Figure 2, A and B, a typical glomerular evoked field potential has a presynaptic fiber volley (FV, abolished by TTX $[0.5 \mu \mathrm{M}]$, but not the cocktail of NBQX [20 $\mu \mathrm{M}]$ and D-APV $[50 \mu \mathrm{M}]$ ); a population spike (pSpike) that is most likely due to action potentials generated in populations of periglomerular cells by ON stimulation (abolished by NBQX); and a field EPSP (fEPSP) that has both AMPA (abolished by NBQX) and NMDA (abolished by D-APV) components (Aroniadou-Anderjaska et al. 1997; Mutoh et al. 2005). Incrementing ON stimulations resulted in incrementing pre- and post-synaptic responses of both AMPA (recorded in aCSF) and NMDA (recorded in NBQX) (Fig. 2C) components.

AMPA I/O relationships were constructed by comparing the sizes of the fiber volley (FV) with the slopes of fEPSPs recorded in aCSF. Figure $2 \mathrm{~A}$ shows that adding D-APV $(50 \mu \mathrm{M})$ to the bath suppressed a late component of the fEPSP and moderately decreased the peak of the fEPSP, while the initial slope of the fEPSP 
A
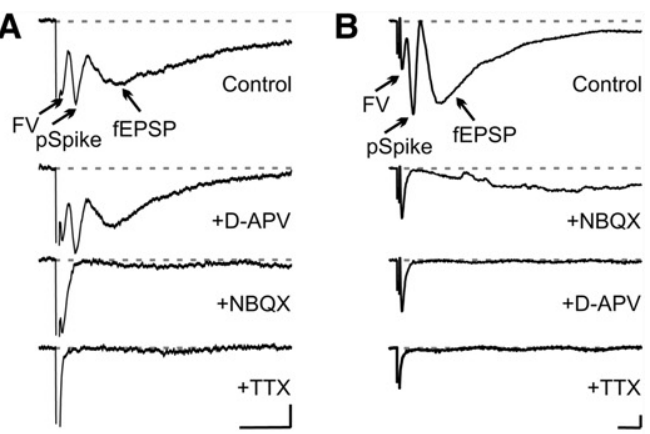

C
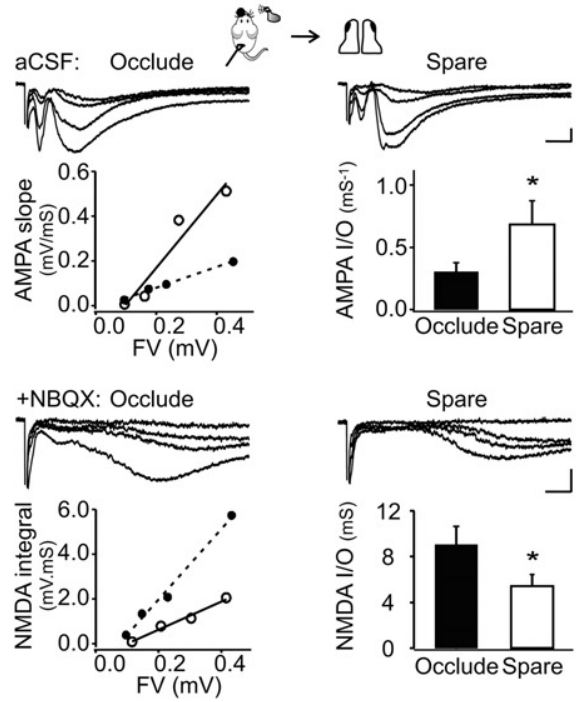

D

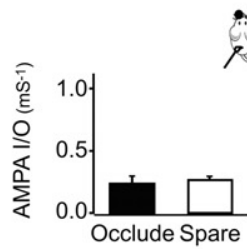

$i^{2} \rightarrow$

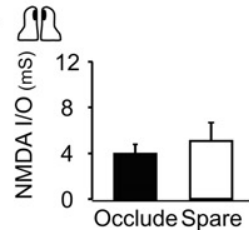

E

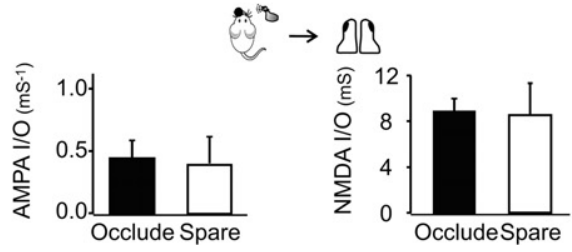

Figure 2. Unilateral occlusion during odor-preference training results in changes of AMPAR and NMDAR only at the peppermint "hotspot" in the non-occluded "learning" olfactory bulb. $(A, B)$ Pharmacological isolation of AMPA and NMDA components of the olfactory nerve evoked potential recorded in the glomerular layer of the olfactory bulb. (FV) Fiber volley, indicating the size of presynaptic activation; (pSpike) population spike of periglomerular neurons (Mutoh et al. 2005); (fEPSP) glomerular field EPSP. Note that the fEPSP has both AMPA and NMDA components, while the pSpike was abolished by an AMPAR antagonist NBQX $(20 \mu \mathrm{M})$. The FV was abolished when TTX $(0.5 \mu \mathrm{M})$ was added to the solution. Scale bars, $0.2 \mathrm{mV}$ and $5 \mathrm{msec}$. $A$ indicates the early D-APV-resistant AMPA component and $B$ indicates the late NBQX-resistant NMDA component. (C) AMPA and NMDA components of fEPSPs measured in the mid-lateral region of paired olfactory bulbs of rat pups $24 \mathrm{~h}$ following Odor + Stroke training (training and recording paradigms as shown in the top cartoon. Mid-lateral recording is indicated by the black spots in the olfactory bulb slices). The pups underwent odor training with unilateral nostril occlusion. Traces are example fEPSPs recorded at various (measured $0.5 \sim 1 \mathrm{mS}$ from the start of the response) was largely unchanged. Recordings from $n=5$ slices showed that the fEPSP peak amplitude decreased from $0.992 \mathrm{mV}$ to $0.85 \mathrm{mV}(14.3 \%$ reduction, $t=2.20, P=0.09$ ). However, the fEPSP slope during D-APV application remained constant $(96.6 \%$ of the fEPSP slope recorded before $\mathrm{D}-\mathrm{APV}$, from $0.412 \mathrm{mV} / \mathrm{mS}$ before $\mathrm{D}-\mathrm{APV}$ to $0.398 \mathrm{mV} / \mathrm{mS}$ during D-APV application. $t=0.93, P=0.40)$. The fEPSP was abolished by the cocktail of NBQX and D-APV, consistent with previous reports showing that the glomerular fEPSP consists of AMPA and NMDA components (Aroniadou-Anderjaska et al. 1997; Mutoh et al. 2005). Therefore, the slope of the fEPSP was used as an index for the AMPA component (Fig. 2C). Application of NBQX itself abolished the early peak of the fEPSP and uncovered a late, D-APV-sensitive component (Fig. 2B). NMDA I/ $\mathrm{O}$ relationships were constructed by comparing FVs with the integral of fEPSPs (area within the $30 \mathrm{mS}$ from the start of fEPSP) recorded in low $\mathrm{Mg}^{2+}$ and NBQX (Fig. 2C). The slopes of the I/O curves were averaged for each group and plotted (Fig. 2C-E). To minimize the variations due to experimental procedures such as the quality of slices and age differences of the animals used (PD6-9), a strict intra-animal comparison was carried out. That is, paired comparisons were made from occluded and spared slices of the same animal. To minimize the potential ON input heterogeneity in different areas of the bulb, only slices at the same cutting plane from the middle portion of each bulb were used for recording and comparison. Data are means \pm SEM. A paired Student's $t$-test was used to determine statistical significance.

Analysis of AMPA and NMDA input-output (I/O) relationships demonstrated both an up-regulation of AMPARs and a down-regulation of NMDARs at the ON synapses in the trained olfactory bulb. The average I/O slope of the AMPA fEPSP slope was $0.69 \pm 0.19 \mathrm{mS}^{-1}$ in spared olfactory bulbs, compared with $0.31 \pm 0.07 \mathrm{mS}^{-1}$ in occluded bulbs $(n=7, t=2.47, P=0.02)$ (Fig. 2C). In contrast, the average I/O slope of the NMDA fEPSP integral was $5.45 \pm 0.97 \mathrm{mS}$ in spared bulbs, compared with $9.04 \pm$ $1.61 \mathrm{mS}$ in occluded bulbs $(n=7, t=2.49, P=0.02)$ (Fig. 2C). The training-induced changes of AMPA and NMDA responses were region specific, as recording from the medial region of the olfactory bulb that underwent the same training did not reveal any significant difference between spared and occluded bulbs (Fig. 2D). The AMPA I/O slopes were $0.27 \pm 0.03 \mathrm{mS}^{-1}$ in spared bulbs and $0.24 \pm 0.06 \mathrm{mS}^{-1}$ in occluded bulbs $(n=5 ; t=0.83, P=0.45)$. The NMDA I/O slopes of the integrals were $5.07 \pm 1.59 \mathrm{mS}$ in spared bulbs and $3.93 \pm 0.83 \mathrm{mS}$ in occluded bulbs $(n=5 ; t=$ $0.77, P=0.48$ ). The effect of a 10-min odor exposure itself was also examined by using the paired olfactory bulbs from the $\mathrm{O} / \mathrm{O}$ LL group. As shown in Figure 2E, odor exposure itself did not change the properties of post-synaptic responses in relation to presynaptic input. On average, there was no significant difference in $\mathrm{I} / \mathrm{O}$ relationships between the spared and occluded bulbs, measured either by the $\mathrm{I} / \mathrm{O}$ relationship of the AMPA slope $\left(0.39 \pm 0.22 \mathrm{mS}^{-1}\right.$ in spared bulbs and $0.44 \pm 0.14 \mathrm{mS}^{-1}$ in

stimulation intensities $(20,40,60,80 \mu \mathrm{A})$ in aCSF (for measuring the AMPA component) and in low $\mathrm{Mg}^{2+}$ aCSF in the presence of NBQX (for isolating the NMDA fEPSP). (Top panels) Example AMPA slope inputoutput curves from the paired olfactory bulbs of one animal (left) and the average from $n=7$ animals (right). ( $($ ) Recordings from the spared bulb. (•) Recordings from the occluded bulb. (Bottom panels) Example of NMDA integral input-output curves from the paired olfactory bulbs of one animal (left) and the average from $n=7$ animals (right). (D) AMPA and NMDA input-output slopes measured in the medial region of the paired olfactory bulbs of rat pups $24 \mathrm{~h}$ following Odor + Stroke training $(n=5)$. (E) AMPA and NMDA input-output slopes measured in the mid-lateral region of paired olfactory bulbs of rat pups $24 \mathrm{~h}$ following Odor Only training $(n=4)$. The pups were exposed to the peppermint odor with unilateral nostril occlusion. 
occluded bulbs; $n=4, t=0.19, P=0.86$ ), or the I/O relationship of the NMDA integral $(8.54 \pm 2.80 \mathrm{mS}$ in spared bulbs and $8.92 \pm$ $1.07 \mathrm{mS}$ in occluded bulbs $n=4, t=0.13, P=0.91$ ).

Unilateral olfactory preference conditioning in PD 6 pups has previously been explored behaviorally in some detail (Kucharski et al. 1986; Kucharski and Hall 1987, 1988). Our behavioral results are consistent with these earlier studies. In one of the earliest reports, Kucharski and Hall (1987) expanded the model by using a number of controls to show that neither the condition of occlusion nor the side trained altered the lateralization of the memory. Having confirmed that our behavioral paradigm successfully replicated the lateralized conditioning effect reported in earlier studies, we were able to use the same paradigm to create intra-individual bulbar slice comparisons, permitting a probe of olfactory nerve-elicited AMPA and NMDA events at the same time point following training.

The results, using in vivo training together with ex vivo testing, demonstrate the predicted increase in the AMPA receptor response at the time of 24-h odor preference memory at the olfactory nerve/mitral cell synapse. This result is consistent with the earlier biochemical demonstration of an increase in membraneassociated AMPA receptors in trained olfactory bulbs $24 \mathrm{~h}$ posttraining and with immunohistochemical evidence of increased staining density for AMPA receptor antibodies $24 \mathrm{~h}$ post-training in the glomerular layer, where these olfactory nerve-mitral connections occur (Cui et al. 2011). While the physiological patterns are evaluated statistically on grouped data, it is of interest that each pair tested showed a difference in the same direction. In contrast to the generalized increase in glomerular AMPA immunohistochemistry seen in the Cui et al. (2011) study, the present electrophysiological data suggest the functional increase in AMPA current and concomitant decrease in NMDA current is restricted to the midlateral bulbar region implicated in early peppermint odor preference learning (Johnson and Leon 1996). Further investigation of the specificity of receptor insertion using combined immunohistochemical and electrophysiological approaches would be helpful in clarifying the localization of bulbar memory circuit changes.

With the present training protocol, an odor preference for the trained odor is seen at $24 \mathrm{~h}$, but not at $48 \mathrm{~h}$. In the immunohistochemical data, the increase in glomerular AMPA receptor staining is not seen $48 \mathrm{~h}$ after the training, consistent with behavioral "forgetting" (Cui et al. 2011). It will be of interest to examine AMPA responses electrophysiologically at a 48 -h time point in a future study. Kucharski and Hall (1987) using a variant of the present 10-min training trial with pulsed odor paired with infused milk as the reward, following 24 -h maternal milk deprivation, which produced odor preferences lasting at least $5 \mathrm{~d}$. Their training paradigm will be useful in asking whether sustained increases in AMPA-mediated connectivity continue to occur during longer term memories.

While it was possible that increased glutamate receptor membrane insertion would be a general phenomenon with training, the NMDA response was decreased, not increased, by training. This suggests a memory-associated decrease in synaptic plasticity $24 \mathrm{~h}$ following training and is consistent with earlier reports of experientially induced down-regulation of NMDA currents (Isaac et al. 1997; Franks and Isaacson 2005). The decrease in NMDA currents may contribute to memory stability and may also reflect the hypothesized operation of bidirectional synaptic plasticity (Bear 2003; Smith et al. 2009). If functionally significant, it should be more difficult to train a different response to peppermint odor at $24 \mathrm{~h}$. Such behavioral experiments have not been attempted. In extended nostril occlusion studies, the NMDA response in the piriform cortex did not decrease with age, as normally seen, and the effect was interpreted as relating to an experientially driven decrease in plasticity during development (Franks and Isaacson 2005). Here, the brief 10-min occlusion period given to all pups was not sufficient to reveal an experiential effect in the odor-only group, which suggests that learning conditions more strongly drive the decrease in NMDA responses than simple odor exposure.

The use of single nostril training provides a strong experimental paradigm for a number of questions about the biology of odor-preference memory. This model will be useful for examining differences in bulbar protein expression for the olfactory bulbs that support the behavioral and physiological changes indicative of memory. In other experiments it may be possible to probe the contribution of the bulbar network change to behavioral memory in the same individuals. To what degree early local network changes in connectivity account for the expressed strength of an odor preference, and to what degree the duration of memory and the duration of physiological changes co-vary, can be addressed with this approach. The present data show directly, for the first time, that the physiological AMPA response is increased $24 \mathrm{~h}$ later at olfactory nerve/mitral cell synapses in olfactory bulbs that have undergone training. The data are consistent with a dual model in which informational connectivity strength increases and plasticity potential decreases with learning (Bear 2003; Smith et al. 2009).

\section{Acknowledgments}

We acknowledge CIHR funding (MOP-102624) to Q.Y., which made this work possible.

\section{References}

Aroniadou-Anderjaska V, Ennis M, Shipley MT. 1997. Glomerular synaptic responses to olfactory nerve input in rat olfactory bulb slices. Neuroscience 79: 425-434.

Bear MF. 2003. Bidirectional synaptic plasticity: From theory to reality. Philos Trans R Soc Lond B Biol Sci 358: 649-655.

Cain DP. 1997. LTP, NMDA, genes and learning. Curr Opin Neurobiol 7: $235-242$.

Cui W, Darby-King A, Grimes MT, Howland JG, Wang YT, McLean JH, Harley CW. 2011. Odor preference learning and memory modify GluA1 phosphorylation and GluA1 distribution in the neonate rat olfactory bulb: Testing the AMPA receptor hypothesis in an appetitive learning model. Learn Mem 18: 283-291.

Debanne D. 1996. Associative synaptic plasticity in hippocampus and visual cortex: Cellular mechanisms and functional implications. Rev Neurosci 7: 29-46.

Franks KM, Isaacson JS. 2005. Synapse-specific downregulation of NMDA receptors by early experience: A critical period for plasticity of sensory input to olfactory cortex. Neuron 47: 101-114.

Isaac JT, Crair MC, Nicoll RA, Malenka RC. 1997. Silent synapses during development of thalamocortical inputs. Neuron 18: 269-280.

Johnson BA, Leon M. 1996. Spatial distribution of [14C]2-deoxyglucose uptake in the glomerular layer of the rat olfactory bulb following early odor preference learning. J Comp Neurol 376: 557-566.

Kucharski D, Hall WG. 1987. New routes to early memories. Science 238: $786-788$.

Kucharski D, Hall WG. 1988. Developmental change in the access to olfactory memories. Behav Neurosci 102: 340-348.

Kucharski D, Johanson IB, Hall WG. 1986. Unilateral olfactory conditioning in 6-day-old rat pups. Behav Neural Biol 46: 472-490.

Malinow R, Malenka RC. 2002. AMPA receptor trafficking and synaptic plasticity. Annu Rev Neurosci 25: 103-126.

Mutoh H, Yuan Q, Knopfel T. 2005. Long-term depression at olfactory nerve synapses. J Neurosci 25: 4252-4259.

Philpot BD. 2005. Sniffing out NMDA receptors in the olfactory cortex. Neuron 47: 3-5.

Rumpel S, Hatt H, Gottmann K. 1998. Silent synapses in the developing rat visual cortex: Evidence for postsynaptic expression of synaptic plasticity. J Neurosci 18: 8863-8874.

Smith GB, Heynen AJ, Bear MF. 2009. Bidirectional synaptic mechanisms of ocular dominance plasticity in visual cortex. Philos Trans R Soc Lond B Biol Sci 364: 357-367.

Received November 14, 2011; accepted in revised form December 9, 2011. 


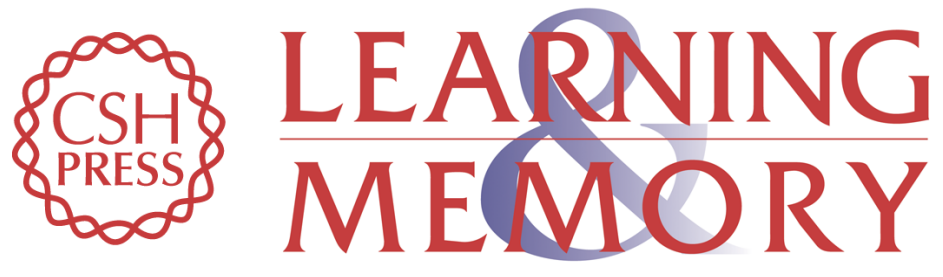

\section{What a nostril knows: Olfactory nerve-evoked AMPA responses increase while NMDA responses decrease at 24-h post-training for lateralized odor preference memory in neonate rat}

Qi Yuan and Carolyn W. Harley

Learn. Mem. 2012, 19:

Access the most recent version at doi:10.1101//m.024844.111

References This article cites 16 articles, 4 of which can be accessed free at: http://learnmem.cshlp.org/content/19/2/50.full.html\#ref-list-1

License

Email Alerting

Receive free email alerts when new articles cite this article - sign up in the box at the Service top right corner of the article or click here. 